\title{
Congestive Heart Failure During Osimertinib Treatment for Epidermal Growth Factor Receptor (EGFR)-mutant Non-small Cell Lung Cancer (NSCLC)
}

\author{
Hiromi Watanabe ${ }^{1}$, Eiki Ichihara ${ }^{2}$, Hirohisa Kano ${ }^{1}$, Kiichiro Ninomiya ${ }^{2}$, \\ Mitsune Tanimoto ${ }^{2}$ and Katsuyuki Kiura ${ }^{1}$
}

\begin{abstract}
:
We herein report a case of congestive heart failure which developed during osimertinib treatment. A 78year-old woman presented with mild exertional dyspnea three weeks after starting osimertinib for the treatment of epidermal growth factor receptor (EGFR) T790M-positive non-small cell lung cancer. She was diagnosed with congestive heart failure caused by the osimertinib. In contrast to trastuzumab, a human epidermal growth factor receptor 2 (HER2) monoclonal antibody that often causes cardiac dysfunction, the causal relationship between osimertinib and cardiotoxicity has so far received little attention and thus remains unclear. However, it inhibits HER2 in addition to mutant EGFR, thereby potentially causing cardiotoxicity.
\end{abstract}

Key words: heart failure, osimertinib, T790M, lung cancer

(Intern Med 56: 2195-2197, 2017)

(DOI: 10.2169/internalmedicine.8344-16)

\section{Introduction}

Dramatic clinical responses to epidermal growth factor receptor (EGFR) tyrosine kinase inhibitors (TKIs) have been observed in non-small cell lung cancers (NSCLCs) harboring EGFR activating mutations. First or second generation EGFR TKIs such as erlotinib and afatinib have become standard therapy for the treatment of EGFR mutated NSCLCs (1). Unfortunately, their efficacy lasts for a relatively short period due to acquired resistance, and more than half of such resistance is caused by T790M, a secondary EGFR mutation. Third generation EGFR TKIs such as osimertinib that inhibit mutant EGFR regardless of the T790 status have been developed (2). Osimertinib was just recently approved in Japan, Europe, and the United States, and it shows a promising activity for the treatment of T790M-positive NSCLC patients, with a $61 \%$ response rate and a 9.6-month progression-free survival (3). This agent is generally well tolerated and less toxic since it does not affect wild type EGFR; however, we still need to pay atten- tion to the adverse effects associated with osimertinib treatment. We herein report a case of congestive heart failure that developed in a patient receiving osimertinib treatment.

\section{Case Report}

A 78-year-old woman was diagnosed in 2011 with metastatic lung adenocarcinoma harboring an EGFR exon21 L858R point mutation. The patient received four regimens of treatment including erlotinib+bevacizumab and platinum doublet chemotherapy. Re-biopsy of the primary lung tumor at the point of progression to erlotinib+bevacizumab revealed an EGFR secondary mutation T790M. She started treatment with osimertinib as the fifth-line regimen in 2016. Three weeks after osimertinib initiation, she presented with face and lower limb edema and mild exertional dyspnea accompanying decreased oxygen saturation (94\% at room air). A chest X-ray showed an enlargement of the cardiac silhouette (Fig. 1). Computed tomography showed bilateral pleural effusion and thickening of bronchovascular bundles, typical findings of pulmonary congestion (Fig. 2). The brain natriu-

${ }^{1}$ Department of Allergy and Respiratory Medicine, Okayama University Hospital, Japan and ${ }^{2}$ Department of Hematology, Oncology, and Respiratory Medicine, Okayama University Graduate School of Medicine, Dentistry and Pharmaceutical Sciences, Japan

Received: October 2, 2016; Accepted: January 10, 2017; Advance Publication by J-STAGE: August 1, 2017

Correspondence to Dr. Eiki Ichihara, ichiha-e@md.okayama-u.ac.jp 


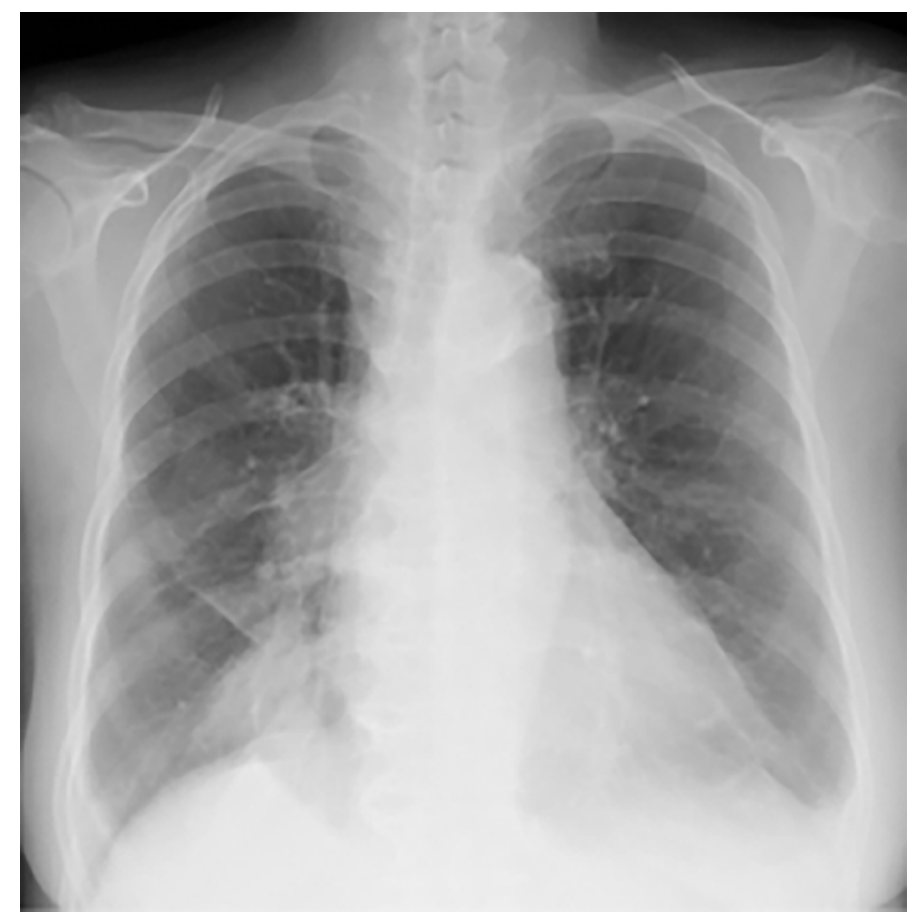

Figure 1. Chest X-ray showing enlargement of the cardiac silhouette.

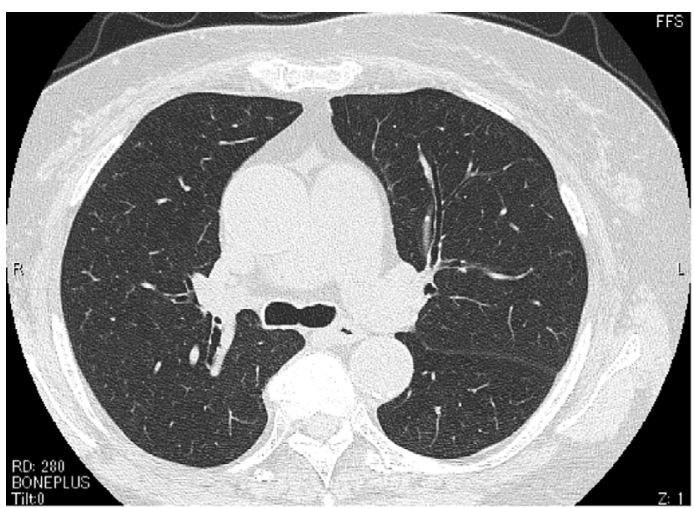

before osimertinib

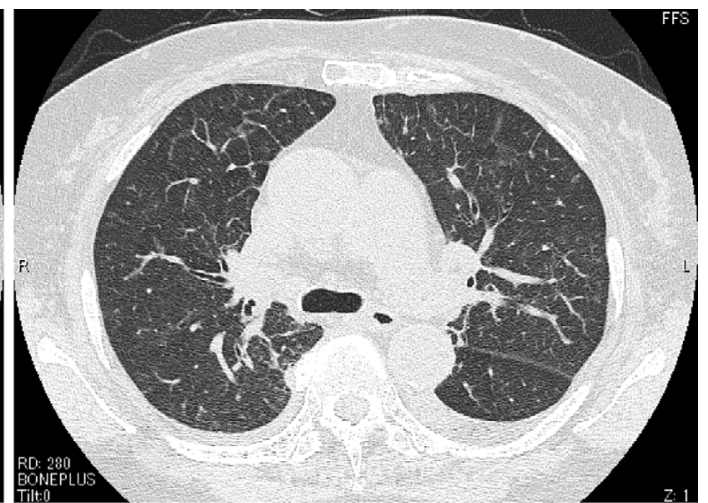

3 weeks after osimertinib

Figure 2. Computed tomography showed the presence of pulmonary congestion. Left: before osimertinib treatment, right: 3 weeks after osimertinib treatment.

retic peptide levels were elevated to $224 \mathrm{pg} / \mathrm{mL}$, from $61 \mathrm{pg} /$ $\mathrm{mL}$ before osimertinib treatment. The creatine kinase $\mathrm{MB}$ isozyme was not elevated, thus ruling out myocardial infarction. Echocardiography showed left atrial dilatation without any wall motion asynergy. The cardiac electrogram findings were normal. Based on these findings, she was diagnosed with congestive heart failure. There was no possible cause other than the osimertinib treatment. She recovered within 5 days after the cessation of osimertinib treatment and the administration of a diuretic. She was able to stop the diuretic without any recurrence of heart failure.

\section{Discussion}

According to the Japan drug fact sheet for osimertinib, cardiovascular complications, such as a decreased ejection fraction, palpitation and chest pain have developed in less than $1 \%$ of osimertinib-treated patients. However, the causal relationship between osimertinib and those complications remains unclear in those cases. Furthermore, there are no precise reports on the occurrence of congestive heart failure during osimertinib. Therefore, this is the first specific report of congestive heart failure induced by osimertinib treatment.

We first suspected that the heart failure may have been caused by ischemic heart disease, since bevacizumab, which 
can cause cardiovascular diseases (4), had been administered to this patient in the past. However, echocardiography and other examinations ruled out the presence of ischemic heart disease. A blood examination also ruled out any viral infection that could have caused myocarditis (data not shown). Furthermore, she had taken no medications associated with cardiotoxicity.

Taken together, we concluded that the heart failure had been caused by osimertinib.

Anthracyclines are the most highly studied cardiotoxic agents, and their dosage is limited due to their cardiotoxicity. Trastuzumab, a monoclonal antibody against human epidermal growth factor receptor 2 (HER2), is another wellstudied cardiotoxic agent and the likelihood of cardiac toxicity was 2.45-fold higher after trastzuzumab (5). Based on experiences of trastuzumab-induced cardiotoxicity, many studies have been performed and have revealed that HER2 signaling is essential for maintaining the heart function (6). Similar to trastuzumab, osimertinib inhibits HER2, thereby potentially causing heart dysfunction (2).

Cardiotoxicity induced by anticancer treatment is increasing in importance as metastatic cancer patients today survive longer. Unfortunately, the cardiotoxic risk in lung cancer patients seems to be underestimated because the prognosis of advanced lung cancer was relatively short, and highly cardiotoxic agents such as anthracyclines and trastuzumab are not usually used. However, the 5-year overall survival rate of metastatic EGFR mutant lung cancer has now reached around $20 \%$ (7). This survival rate was calculated before the introduction of osimertinib, so the latest survival rates could thus now be even longer. In addition, we now have potentially cardiotoxic agents including osimeritinib and afatinib, both of which inhibit HER2.

In conclusion, we experienced a case of congestive heart failure which developed while the patient was administered osimertinib treatment, thus showing the need for a careful follow-up of the cardiac function during osimertinib treat- ment.

Author's disclosure of potential Conflicts of Interest (COI). Mitsune Tanimoto: Honoraria, Daiichi-Sankyo Pharmaceutical, Chugai Pharmaceutical, Taiho Pharmaceutical, MSD, Pfizer and Boehringer Ingelheim.

Katsuyuki Kiura: Honoraria, Eli Lilly Japan, Nihon Kayaku, AstraZeneca, Daiichi-Sankyo Pharmaceutical, Chugai Pharmaceutical, Taiho Pharmaceutical, and Sanofi-Aventis.

\section{References}

1. Yang JC, Wu YL, Schuler M, et al. Afatinib versus cisplatin-based chemotherapy for EGFR mutation-positive lung adenocarcinoma (LUX-Lung 3 and LUX-Lung 6): analysis of overall survival data from two randomised, phase 3 trials. Lancet Oncol 16: 141-151, 2015.

2. Cross DA, Ashton SE, Ghiorghiu S, et al. AZD9291, an irreversible EGFR TKI, overcomes T790M-mediated resistance to EGFR inhibitors in lung cancer. Cancer Discov 4: 1046-1061, 2014.

3. Janne PA, Yang JC, Kim DW, et al. AZD9291 in EGFR inhibitorresistant non-small-cell lung cancer. N Engl J Med 372: 16891699, 2015.

4. Economopoulou P, Kotsakis A, Kapiris I, Kentepozidis N. Cancer therapy and cardiovascular risk: focus on bevacizumab. Cancer Manag Res 7: 133-143, 2015.

5. Viani GA, Afonso SL, Stefano EJ, De Fendi LI, Soares FV. Adjuvant trastuzumab in the treatment of her-2-positive early breast cancer: a meta-analysis of published randomized trials. BMC Cancer 7: 153, 2007.

6. Balduzzi S, Mantarro S, Guarneri V, et al. Trastuzumab-containing regimens for metastatic breast cancer. Cochrane Database Syst Rev 6: CD006242, 2014.

7. Lin JJ, Cardarella S, Lydon CA, et al. Five-year survival in EGFR-mutant metastatic lung adenocarcinoma treated with EGFRTKIs. J Thoracic Oncol 11: 556-565, 2016.

The Internal Medicine is an Open Access article distributed under the Creative Commons Attribution-NonCommercial-NoDerivatives 4.0 International License. To view the details of this license, please visit (https://creativecommons.org/licenses/ by-nc-nd/4.0/).

(C) 2017 The Japanese Society of Internal Medicine Intern Med 56: 2195-2197, 2017 